\title{
Why the entropy of spacetime is independent of species of particles: the species problem
}

\author{
Yu-Zhu Chen ${ }^{1}$, Wen-Du Li ${ }^{1,2}$, Wu-Sheng Dai ${ }^{1, \mathrm{a}}$ \\ ${ }^{1}$ Department of Physics, Tianjin University, Tianjin 300350, People's Republic of China \\ 2 Theoretical Physics Division, Chern Institute of Mathematics, Nankai University, Tianjin 300071, People's Republic of China
}

Received: 8 September 2016 / Accepted: 30 July 2018 / Published online: 9 August 2018

(C) The Author(s) 2018

\begin{abstract}
The Hawking radiation emits all species of particles, but the Bekenstein-Hawking entropy is independent of the number of the species of particles. This is the so-called species problem-a puzzling problem for a long time. In this paper, we suggest a solution to this problem. A result of the scheme is that the black hole atmosphere has a mass equaling 3/8 mass of a classical Schwarzschild black hole, which agrees with 't Hooft's brick wall model.
\end{abstract}

By only taking a scalar field into account, one can obtain the whole Bekenstein-Hawking entropy [1,2]. Nevertheless, the Hawking radiation emits all species of particles. What is the role of other species of particles. It is hard to believe that only one species of particle contributes to the entropy. This is the so called species problem $[3,4]$.

In this paper, we suggest a solution to the species problem. A result of our scheme is that the entropy of a spacetime is independent of the number of species of particles. This means that even there are more than one species of particles, even there exists some unknown species of particles, the entropy of a spacetime is still the Bekenstein-Hawking entropy, as same as that calculated from one single scalar field.

In our scheme, the black hole entropy is regarded as the entropy of the atmosphere outside the horizon. The reason why the entropy of a spacetime can be regarded as the entropy of its atmosphere is that the entropy of the atmosphere is the entanglement entropy [5-7], and the entropy of a black hole can also be regarded as the entanglement entropy [8-11]. More concretely, the entropy of the atmosphere was first calculated by t' Hooft [1] in the brick wall model, and thereafter some authors pointed out that the entropy of the atmosphere should be regarded as the entanglement entropy [5-7]. In fact, the calculation of the entropy of a black hole is the same as the entanglement entropy calculation [5]. The species problem then arises in the entanglement entropy calculation.

\footnotetext{
a e-mail: daiwusheng@tju.edu.cn
}

The species problem has a direct bearing on the understanding of the spacetime entropy. Much effort has been devoted to understanding the species problem. The simplest scheme suggests that only a particular field contributes to the spacetime entropy $[2,3]$. Based on the Euclidean path integral method, Jacobson finds that the leading contribution to the entropy in the effective action is the classical HilbertEinstein term $\hbar a_{1} \int d^{4} x \sqrt{g} R$; by regarding the expansion coefficient $\hbar a_{1}$ as the renormalized inverse gravitational constant $1 /(16 \pi G)$, the leading contribution is the same as the Bekenstein-Hawking entropy $[3,12]$. Therefore, the entropy calculated by the Euclidean path integral is independent of the number of species of particles. Frolov comes to a same conclusion by a particular method [13], who demonstrates that the one-loop contribution to the entropy comparing with the tree-level contribution is ignorable. A more extensively used scheme is to correct the cutoff. When regarding the spacetime entropy as the entanglement entropy [4,5], a short distance cutoff $\Lambda$ must be brought in $[1,10,14]$. By resetting the cutoff to $\Lambda / \sqrt{N}$, where $N$ is the number of species of particles, the entanglement entropy contributed by all species of particles adds up to the Bekenstein-Hawking entropy [15]. Susskind suggests a similar method, in which the gravitational constant $G$ is renormalized to obtain the Bekenstein-Hawking entropy [8]. More discussions on the cutoff approach are also given by other authors [15-17].

Our scheme is as follows.

Where there is a horizon there is an atmosphere. The existence of the Hawking radiation ensures that a spacetime with a horizon must have an atmosphere consisting of the particles which are emitted out of the horizon and fall back into the horizon in a dynamic equilibrium.

That is, a spacetime indeed consists of two indivisible parts: a bare spacetime which is a classical spacetime solved from the Einstein equation and its atmosphere which is a pure quantum effect. In other words, when talking about a 
spacetime, we must simultaneously consider both the classical bare spacetime and its atmosphere. These two parts are always concomitant with each other from the birth of the spacetime. In our scheme, the bare spacetime and its atmosphere are regarded as a single whole system.

A spacetime with an atmosphere is indeed a dressed space consists of a classical bare spacetime and a quantum atmosphere, just like a dressed particle - a bare particle with a virtual-particle cloud. Nevertheless, it should be emphasized that rather than the virtual-particle cloud of a particle, the atmosphere of a spacetime consists of real particles.

The entropy of a spacetime all comes from its atmosphere. Since a spacetime is indeed a classical bare spacetime with its atmosphere, the entropy of a spacetime is then the sum of the entropy of the bare spacetime and the entropy of the atmosphere:

$S=S_{\text {bare }}+S_{\text {atmosphere }}$.

The entropy $S=\ln \Omega$ with $\Omega$ the number of states. The bare spacetime is regarded as a mechanical system and has only one certain state, so it does not contribute to the entropy, i.e., $S_{\text {bare }}=\ln 1=0$. Consequently, the atmosphere contributes all the entropy of a spacetime: $S=S_{\text {atmosphere }}$.

In a word, the entropy of a spacetime is just the entropy of the atmosphere of the spacetime.

All species of particles near the horizon behave like massless particles. The Hawking radiation emits all species of particles, massive and massless. Nevertheless, all species of particles near the horizon behave like massless particles.

We take a scalar particle near the horizon of a Schwarzschild spacetime as an example to illustrate this.

The radial Klein-Gordon equation in a Schwarzschild spacetime with the tortoise coordinate $r_{*}=$ $r+r_{0} \ln \left(r / r_{0}-1\right)$ reads [7]

$$
\left\{-\frac{\partial^{2}}{\partial t^{2}}+\frac{\partial^{2}}{\partial r_{*}^{2}}-\left(1-\frac{r_{0}}{r}\right)\left[\frac{r_{0}}{r^{3}}-\frac{l(l+1)}{r^{2}}+m^{2}\right]\right\} R(t, r)=0,
$$

where $r_{0}=2 M$ is the horizon radius with $M$ the mass of the classical bare spacetime. Near the horizon, $r \rightarrow r_{0}$, the massive equation, Eq. (0.2), reduces to a massless equation:

$$
\left(-\frac{\partial^{2}}{\partial t^{2}}+\frac{\partial^{2}}{\partial r_{*}^{2}}\right) R(t, r)=0
$$

This shows that even a massive particle still has a behavior like a massless particle near the horizon.

The entropy of any species of massless particles has the same relation to the internal energy. Near the horizon, all particles behave like massless particles. It can be shown that for both massless bosonic and massless fermionic gases, i.e., ultrarelativistic bosonic and fermionic gases, the relation between the entropy $S$ and the internal energy $U$ is [18]

$S=\frac{4}{3} \beta U$.

It should be emphasized that near the horizon of the Schwarzschild spacetime, the spacetime is almost flat [7]. This allows us to apply the result of statistical mechanics in flat spacetime directly.

The spacetime entropy is independent of the species of particles. Now, it is ready to describe our scheme.

It is already shown that no matter what species of particles are, bosonic or fermionic, massive or massless, the entropies are always have the same relation to the energies. Suppose that there are $N_{B}$ species of bosons and $N_{F}$ species of fermions. The total energy of the atmosphere is, of course,

$U=\sum_{i=1}^{N_{B}} U_{i}^{\text {Bose }}+\sum_{i=1}^{N_{F}} U_{i}^{\text {Fermi }}$

The entropy of the system, by eq. (0.4), then reads

$$
\begin{aligned}
S & =\sum_{i=1}^{N_{B}} S_{i}^{\text {Bose }}+\sum_{i=1}^{N_{F}} S_{i}^{\text {Fermi }} \\
& =\sum_{i=1}^{N_{B}} \frac{4}{3} \beta U_{i}^{\text {Bose }}+\sum_{i=1}^{N_{F}} \frac{4}{3} \beta U_{i}^{\text {Fermi }} \\
& =\frac{4}{3} \beta U .
\end{aligned}
$$

This result tells us that no matter how many species of particles are emitted, the entropy depends only on the total energy of particles rather than the number of the species of particles.

The atmosphere accounts for $3 / 11$ of the mass of a Schwarzschild black hole. For the Schwarzschild spacetime, we can arrive at the Bekenstein-Hawking entropy

$S=4 \pi M^{2}$

so long as the total energy of the atmosphere is

$U=\frac{3}{8} M$.

Note that for the Schwarzschild spacetime the reciprocal of the temperature $\beta=8 \pi M$.

Since the mass of the total system, $m_{\text {total }}$, in our scheme is the bare mass, $m_{\text {bare }}=M$, plus the mass of the atmosphere, $m_{\text {atmosphere }}=\frac{3}{8} M$, the ratio of the bare mass and the mass of the atmosphere is $m_{\text {bare }}: m_{\text {atmosphere }}=8: 3$, or, $m_{\text {bare }}$ : $m_{\text {total }}=8: 11$ and $m_{\text {atmosphere }}: m_{\text {total }}=3: 11$.

In a word, in this scheme the entropy is independent of the species of particles.

The key point of this scheme is to regard the atmosphere of a spacetime, which is a pure quantum effect, as a part of 
the spacetime. In the scheme, a spacetime consists of two parts: the classical bare spacetime and its atmosphere. The entropy of the spacetime all comes from the atmosphere. Considering that any particle near the horizon behaves like a massless particle, we conclude that the atmosphere can be considered as ultra-relativistic gases. As a result, the entropy, regardless of the species of particles, is proportional to the energy (mass) and is independent of the species of particles.

Furthermore, since a spacetime is divided into a classical part (the solution of the Einstein equation) and a quantum part (the atmosphere), when studying the quantum properties of a spacetime, one can only focus on the atmosphere of the spacetime. That is to say, the property of a thermal field in a spacetime background [19] reflects the quantum behavior of the spacetime.

A by-product of this scheme is that for a Schwarzschild black, the ratio of the mass of the classical bare black hole and its atmosphere is 8:3. Based on the brick wall model, 't Hooft also showed that the energy of the bosonic gas outside the Schwarzschild horizon is $\frac{3}{8} M[1]$. Our result agrees with 't Hooft's result.

Finally, inspired by the hierarchy problem, we would like to suggest an alternative scheme to understand the species problem. In a series of works [15,20-24], the authors suggest a bound on the fundamental scale of the spacetime imposed by the species of the particles in black hole physics. A modified Planck length $l_{\text {species }}=\sqrt{N_{\text {species }}} l_{p}$ is introduced, where $l_{p}$ is the Planck length and $N_{\text {species }}$ is the number of the species of particles in the world [24]. The BekensteinHawking entropy of a black hole is $S_{B H}=\frac{1}{4} k_{B} A / l_{p}^{2}$. The entropy contributed by one certain species of particles is $S_{\text {species }}=\frac{1}{4} k_{B} A / l_{\text {species }}^{2}$, in which the Planck constant $l_{p}$ is replaced by the modified Planck length $l_{\text {species }}$. If there are $N$ species of particles in the world, the entropy of a black hole is the sum of the contributions of all species of particles. We then have $S_{B H}=N S_{\text {species }}=\frac{1}{4} k_{B} A / l_{p}^{2}$. This result tells us that despite how many species of particles in the world, the entropy of a black hole is still the Bekenstein-Hawking entropy. In other words, the entropy is independent of the species of particles.

Acknowledgements We are very indebted to Dr G. Zeitrauman for his encouragement. This work is supported in part by NSF of China under Grant nos. 11575125 and 11675119.

Open Access This article is distributed under the terms of the Creative Commons Attribution 4.0 International License (http://creativecomm ons.org/licenses/by/4.0/), which permits unrestricted use, distribution, and reproduction in any medium, provided you give appropriate credit to the original author(s) and the source, provide a link to the Creative Commons license, and indicate if changes were made.

Funded by SCOAP ${ }^{3}$.

\section{References}

1. G. Hooft, On the quantum structure of a black hole. Nucl. Phys. B 256, 727-745 (1985)

2. S.N. Solodukhin, One-loop renormalization of black hole entropy due to nonminimally coupled matter. Phys. Rev. D 52(12), 7046 (1995)

3. T. Jacobson, Black Hole Entropy and Induced Gravity. arXiv:gr-qc/9404039 (1994) (arXiv preprint)

4. J.D. Bekenstein, Do We Understand Black Hole Entropy? arXiv:gr-qc/9409015 (1994) (arXiv preprint)

5. R.M. Wald, The thermodynamics of black holes. In: Advances in the Interplay Between Quantum and Gravity Physics (Springer, 2002), pp. 477-522

6. S. Mukohyama, Aspects of Black Hole Entropy. arXiv:gr-qc/9912103 (1999) (arXiv preprint)

7. L. Susskind, J. Lindesay, An Introduction to Black Holes, Information and the String Theory Revolution (World Scientific, Singapore, 2005)

8. L. Susskind, J. Uglum, Black hole entropy in canonical quantum gravity and superstring theory. Phys. Rev. D 50(4), 2700 (1994)

9. J.S. Dowker, Remarks on geometric entropy. Class. Quant. Gravity 11(4), L55 (1994)

10. L. Bombelli, R.K. Koul, J. Lee, R.D. Sorkin, Quantum source of entropy for black holes. Phys. Rev. D 34(2), 373 (1986)

11. C. Callan, F. Wilczek, On geometric entropy. Phys. Lett. B 333(1), 55-61 (1994)

12. F. Larsen, F. Wilczek, Renormalization of black hole entropy and of the gravitational coupling constant. Nucl. Phys. B 458(1), 249-266 (1996)

13. V.P. Frolov, Why the entropy of a black hole is a/4. Phys. Rev. Lett. 74(17), 3319 (1995)

14. V. Frolov, I. Novikov, Dynamical origin of the entropy of a black hole. Phys. Rev. D 48(10), 4545 (1993)

15. G. Dvali, S.N. Solodukhin, Black Hole Entropy and Gavity Cutoff. arXiv:0806.3976 (2008) (arXiv preprint)

16. J.-G. Demers, R. Lafrance, R.C. Myers, Black hole entropy without brick walls. Phys. Rev. D 52(4), 2245 (1995)

17. T. Jacobson, R. Parentani, Black hole entanglement entropy regularized in a freely falling frame. Phys. Rev. D 76(2), 024006 (2007)

18. R. Pathria, P. Beale, Statistical Mechanics (Elsevier Science, Oxford, 2011)

19. W.-S. Dai, M. Xie, An approach for the calculation of one-loop effective actions, vacuum energies, and spectral counting functions. J. High Energy Phys. 2010(6), 1-29 (2010)

20. N. Arkani-Hamed, S. Dimopoulos, G. Dvali, The hierarchy problem and new dimensions at a millimeter. Phys. Lett. B 429(3), 263-272 (1998)

21. G. Dvali, Black holes and large $\mathrm{n}$ species solution to the hierarchy problem. Fortschr. Phys. 58(6), 528-536 (2010)

22. G. Dvali, C. Gomez, Black hole's 1/n hair. Phys. Lett. B 719(4), 419-423 (2013)

23. G. Dvali, C. Gomez, Quantum information and gravity cutoff in theories with species. Phys. Lett. B 674(4), 303-307 (2009)

24. G. Dvali, C. Gomez, D. Lüst, Black hole quantum mechanics in the presence of species. Fortschr. Phys. 61(7-8), 768-778 (2013) 